\title{
Effects of customary drugs and modified herbal hypoglycemic therapy on blood glucose and total antioxidant capacity in Streptozotocin induced diabetes in albino rats: A comparative analysis
}

\author{
${ }^{1 *}$ Iftikhar Ahmad, ${ }^{2}$ Hafeez ul Hassan, ${ }^{3}$ Faisal Ahmed, ${ }^{4}$ Muhammad Sarwar, ${ }^{5}$ Kefi Iqbal \\ ${ }^{1}$ Department of Biochemistry, Baqai Medical University, Karachi \\ ${ }^{2}$ Department of Physiology, Baqai Medical University, Karachi \\ ${ }^{3}$ Department of Family Medicine, Aga Khan University Hospital, Karachi \\ ${ }^{4}$ Department of Chemical Pathology, Al Jouf Medical College, Al Jouf, Kingdom of Saudi Arabia \\ ${ }^{5}$ Department of Dental Materials, Baqai Medical University, Karachi \\ *Corresponding author E-mail: driftikhaahmed68@gmail.com
}

\begin{abstract}
Back ground:

Evidences suggest that oxidative stress may play an important role in the etiology of diabetes and diabetic complications. The free radicals can be encountered by the conventional treatment of diabetes. Alternatively, some plants and trace elements may be proven to bring desirable results including Coriander and Vanadyl sulfate. To produce hypoglycemic and anti oxidative effects, expensive and huge amount of these are required.

Objective:

An innovative method should be developed in which the Vanadyl sulfate may be up taken by coriander so that it must render it non toxic to produce hypoglycemic and anti oxidative effects in diabetic rats.

Material \& methods:

150 albino rats were employed. 25 rats were kept normal (Group A). 125 made diabetic by STZ injection. Total 05 groups were identified and developed; each comprised of 25 rats. These were treated by Vanadyl sulfate (Group B), coriander only (Group C), Vanadyl sulfate up taken by coriander (V+C) (Group D), insulin treated (Group E) and last one did not receive any treatment (Group F).

Results:

The hypoglycemic effects and total antioxidant activity produced by the Vanadyl sulfate uptaken coriander are compatible than that of the insulin with negligible difference. All the other employed groups in the study did not reveal marked hypoglycemic activity. Pharmacological studies of Vanadyl sulfate uptaken herb demonstrated no deleterious effects.

Conclusion:

The detoxification of vanadyl sulfate has been achieved through possible auto oxidation of this valuable trace element by coriander. Vanadyl sulfate uptaken by coriander acts as antidiabetic agent and shows a considerable insulinomimetic and total antioxidant activity in the Diabetic rats.
\end{abstract}

Keywords: Diabetes, vanadyl sulfate, coriander, total antioxidant capacity, insulinomimetic activity

\section{Introduction}

The Diabetes affects up to $6 \%$ of the population in the world [1]. Diabetes currently affects about 200 million people worldwide and is expected to reach 333 million by 2025. Almost $10 \%$ of the adult people of Pakistan suffer from diabetes mellitus [2]. During diabetes, persistent hyperglycemia causes increased production of free radicals especially reactive oxygen species (ROS), for all tissues from glucose auto-oxidation and protein glycosylation. Free radicals are generated as by-products of normal cellular metabolism; however, uncontrolled DM is known the best to disturb the balance between ROS production and cellular defense mechanisms. This imbalance can result in cell dysfunction and destruction resulting in tissue injury. The increase in the level of ROS in diabetes could be due to their increased production and/ or decreased destruction by nonenzymatic antioxidants e.g. Vitamin A, C and $\mathrm{E}$ and enzymatic antioxidants which may include Catalase (CAT), glutathione peroxidase (GSH-Px), and superoxide dismutase (SOD) 
[3]. Therefore, the level of these antioxidant enzymes critically influences the susceptibility of various tissues to oxidative stress and is associated with the development of complications in diabetes. In addition, this is particularly relevant and dangerous for the beta islet, which is among those tissues that have the lowest levels of intrinsic antioxidant defenses. Free radicals may play an important role in the causation and complications of diabetes mellitus. During the course of the disorder, alterations in the endogenous free radical scavenging defense mechanisms may lead to ineffective scavenging of reactive oxygen species, resulting in oxidative damage and tissue injury. Oxidative stress is currently suggested as mechanism underlying diabetes and diabetic complications [4].

In the light of these facts a significant correlation, between total antioxidant capacity and clinical characteristics of diabetic patients including their blood levels of glucose and glycated hemoglobin, suggests that the measurement of total antioxidant capacity in diabetic patients can be a marker of glycaemic control [5].

Free radicals can be encountered by the treatment of diabetes mellitus with insulin and/or oral synthetic hypoglycemics [6]. Though these agents are giving encouraging results, yet they are expensive, painful giving undesirable psychological effects [7]. In addition, they exhibit side/after effects in long-term use. Recent evidence suggests that impaired antioxidant status is involved in oxidative stress associated with diabetes [8].

Some plants and trace elements may be proven the candidates or alternatives of allopathic medicines i.e. insulin, Biguanides and Sulphonyl Ureas, to bring about the promising and desirable results to circumvent the day-by-day spreading menace of diabetes mellitus with minimum or no side effects [9]. In herbal antidiabetic studies, along with a list of herbs, coriander (Coriandrum Sativum) [10] has been reported to be hypoglycemic. A study on mice found that coriander extract had both insulin-releasing and insulin-like activity [11]. Although, an anti-diabetic activity of coriander is already known, yet the amount which exhibit this effect, is a huge amount $(200 \mathrm{mg} / \mathrm{kg} / \mathrm{day} / 15 \mathrm{days})$ is required and not without side effects [12] [13]. Similarly Vanadyl sulfate exhibit the anti diabetic activity yet its toxicity have been observed at high doses $(0.75 \mathrm{mg} / \mathrm{dl} / \mathrm{day} / 17$ days $)$ that were required to produce hypoglycemic effects [14]. Vanadium may act as a co-factor for enzymes involved in blood sugar metabolism, lipid and cholesterol metabolism [15]. Animal experiments have shown that vanadium can mimic the effects of insulin and reduce blood sugar levels from high to normal. These benefits are seen with high doses and there have been limited clinical trials with vanadium salts in patients with Type II diabetes, indicating that vanadium may have therapeutic potential in the treatment of diabetes [16].

In animal research, a very narrow line has been found between the benefits and toxicity of this trace mineral. Reports at some labs shows death rate as high as $50 \%$ at doses required to lower blood sugars [17]. Vanadium has been shown to cause death in both pregnant rats (45\%) and fetuses [18]. In addition, different labs have produced different outcomes related to the blood sugars, with some labs finding little blood sugar lowering effect.

Obvious toxic effects from vanadium compounds are usually seen in lab animals at minimum doses of 2.5 to 7.5 $\mathrm{mg} / \mathrm{kg} / \mathrm{day}$ [19]. This is higher than typical over-the-counter doses of $30 \mathrm{mg}$ to $60 \mathrm{mg}$ per day, [20]. Some researchers speculate that excess vanadium could also be involved in several diseases of the kidney [21] and bone [22] where it is known to accumulate.

In recent years some researchers made efforts to detoxify vanadium or its corresponding salts. One study found that the interaction of sodium metavanadate and $\mathrm{VOCl}_{3}$ with ascorbic acid might be one of the possible natural reducing agents of vanadium. [23].

In another scientific cram the interaction of the $\mathrm{VO}^{2+}$ cation with the sodium salt of 2,3-dimercapto-1-propanesulfonic acid (DMPS) established to be a potentially useful detoxification agent for vanadium [11].

Primary and core purpose of our study is to find such a way that can minimize the toxicity of Vanadyl sulfate suitable for lowering the blood glucose level which may be without undesirable side effects and cost effective.

\section{Materials and methods}

\subsection{Animals}

Wister male albino rats weighing 180-200 grams were used for all the experiments. Animals were kept in the animal house at temperatures of $22-26^{\circ} \mathrm{C}$ and relative humidity of $55 \%$ and acclimatized for a period of 07 days. The animals were fed on standard chow diet and given tap water ad libitum until treatment. All the experiments with animal were approved by the Animal Ethical Committee (AEC) of Baqai Medical University, Karachi. Pakistan.

\subsection{The Coriander:}

Coriander was grown in well drained pots under light shades. Minimum of four hours of sunshine in a day was given for proper growth. The soil in which the coriander was grown is well aerated and drainage. The seeds were sown in 1 $\mathrm{cm}$ deep holes and covered the soil and compost. The coriander was sown in a row around $4 \mathrm{~cm}$ apart and the space 
between the rows was kept at least $35 \mathrm{~cm}$. when the seedlings were about $4-5 \mathrm{~cm}$ these were thin down to about $20 \mathrm{~cm}$ apart. To ensure a sustained crop throughout the season it was sown every few weeks. A liquid fertilizer to the soil was applied with a gap of $2-3$ weeks to promote the growth. The plant was watered every morning, but never over watered to prevent it from rotting. The coriander was grown with or without Vanadyl sulfate in a quantity of $10 \mathrm{Gm} / \mathrm{kg}$ of soil. The Vanadyl sulfate was dissolved in simple tap water and then poured in the soil, after three week of sowing the seedlings; the soil was assessed and estimated for Vanadyl sulfate from PCSIR laboratories, Karachi.

The rats were given maximum amount of the Vanadyl sulfate uptaken Coriandrum Sativum, orally through water and in the food.

\subsection{Chemical analysis and methodology to induce DM through STZ injection}

2.3.1 This induction protocol is used to induce diabetes in experimental studies similar to a type I diabetic by using Streptozotocin (STZ) in calculated dose as it can destroy Beta cell of Pancreas [24].

\subsubsection{Estimation of Total Antioxidant Capacity by FRAP Assay. [25] PRINCIPLE}

The ferric reducing of ability in plasma (FRAP) has been used to estimate the TAC because the reducing ability of metals may be equivalent to Antioxidant power. In this scheme excess $\mathrm{Fe}^{+3}$ is reduced by the rate limiting factor of $\mathrm{Fe}^{+2}$-TPTZ which leads to blue color. The OD was read at $593 \mathrm{~nm}$.

\subsubsection{Estimation of Blood Glucose [26] :}

PRINCIPLE: The enzyme glucose oxidase catalyzes the oxidation of glucose to gluconic acid and hydrogen peroxide $\left(\mathrm{H}_{2} \mathrm{O}_{2}\right)$. The hydrogen peroxide formed in this reaction reacts with 4-aminoantipyrine and phenol in the presence of peroxidase (POD) to form quinoneminie dye in Alcohol solvent. The amount of dye formed is proportional to the glucose concentration.

\subsection{Grouping of the experimental Rats.}

\begin{tabular}{|c|c|c|}
\hline Groups & Dose of drug used as anti diabetic therapy & $\begin{array}{l}\text { Interval of } \\
\text { therapy }\end{array}$ \\
\hline $\begin{array}{l}25 \text { Animals were kept normal and then } \\
\text { grouped as Control }(\mathbf{A}) \text {. }\end{array}$ & No treatment & 21 days \\
\hline $\begin{array}{l}\text { Group B of } 25 \text { rats with Diabetes } \\
\text { (Diabetic rats treated with Vanadyl sulfate }\end{array}$ & $7.5 \mathrm{mg} / \mathrm{ml}$ with $0.5 \%$ sodium chloride to reduce its toxicity & 21 days. \\
\hline $\begin{array}{l}\text { Group C of } \mathbf{2 5} \text { rats with Diabetes } \\
\text { (Diabetic rats treated with coriander only) }\end{array}$ & $\begin{array}{l}\text { The Coriander was administered in Tween solution } \\
\text { through nasogastric tube, at least } 03 \mathrm{ml}-04 \mathrm{ml} / \text { day in three } \\
\text { equally divided doses and maximum in routine feed }\end{array}$ & 21 days \\
\hline $\begin{array}{l}\text { Group D of } \mathbf{2 5} \text { rats with Diabetes } \\
\text { (Diabetic rats treated with Vanadyl sulfate } \\
\text { uptaken Coriander }\end{array}$ & $03 \mathrm{ml}-04 \mathrm{ml} /$ day thrice daily in identical doses, & 21 days \\
\hline $\begin{array}{l}\text { For Group } \mathbf{E} \text { of } 25 \text { rats with Diabetes } \\
\text { (insulin treated) }\end{array}$ & $\begin{array}{l}\text { insulin (Detemir }{ }^{\circledR} \text { ) in a dose of } 2 \mathrm{IU} \text {, was administered once } \\
\text { daily intraperitoneally to diabetic animals (insulin treated) } \\
\text { group, each day }\end{array}$ & 21 days. \\
\hline $\begin{array}{l}\text { Group F of } 25 \text { rats were made Diabetic } \\
\text { with NO treatment and were kept under } \\
\text { observation for their general health. }\end{array}$ & $\begin{array}{l}\text { No treatment Whenever the animal was observed dull or } \\
\text { seemed unconscious it was sacrificed and the blood was } \\
\text { drawn directly from the heart. }\end{array}$ & 21 days \\
\hline
\end{tabular}

**Every week the FBS was determined and then mean value was calculated for each group.

\section{Aims and objectives}

* To give Vanadyl sulfate in a natural environment in order to minimize its toxicity through uptake by a suitable plant. (Coriandrum sativum)

* To analyze the insulinomimetic effects of Vanadyl sulfate uptaken by Coriandrum sativum.

* To investigate the Total antioxidant capacity of Vanadyl sulfate uptaken by Coriandrum sativum in diabetic rats. 


\section{Results}

Table 1: The serum levels of total antioxidant capacity (TAC) of Group A and other groups in the study.

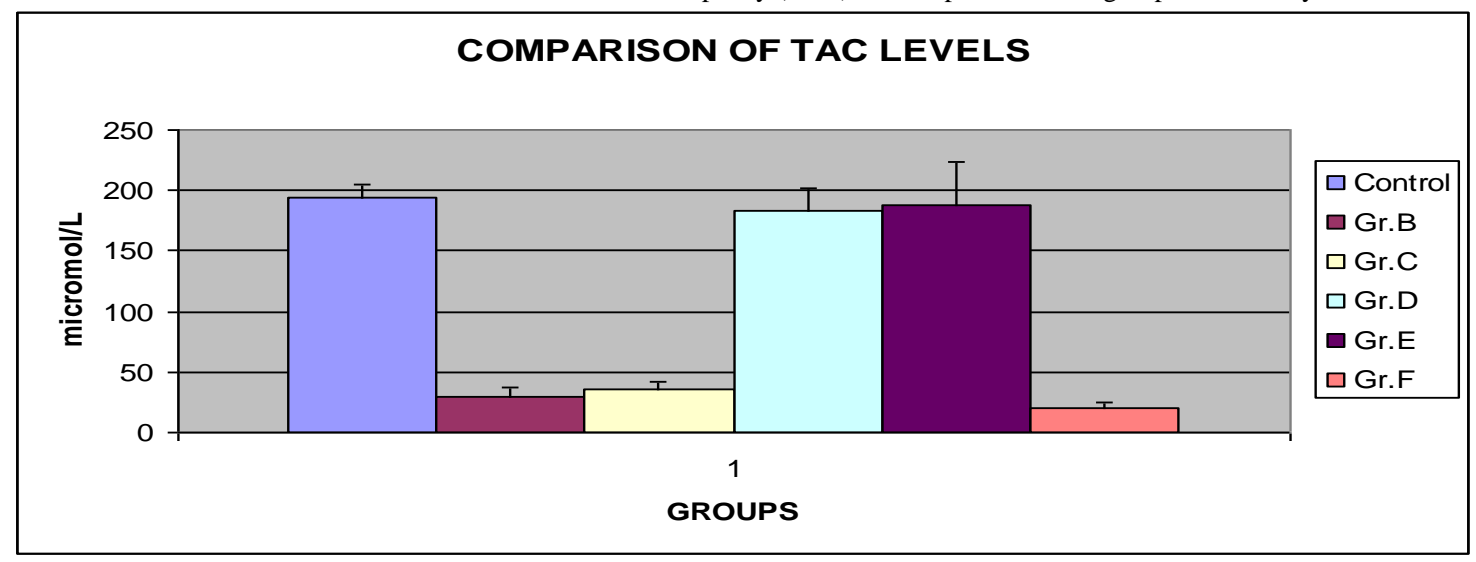

\begin{tabular}{|l|l|l|l|l|l|}
\hline TAC $(\boldsymbol{\mu m o l} / \mathbf{L})$ & & & & & \\
\hline Control & Gr.B & Gr.C & Gr.D & Gr.E & Gr.F \\
\hline $193.88 \pm 11.85$ & $29.55 \pm 6.95$ & $35.23 \pm 6.45$ & $183.54 \pm 18.95$ & $187.78 \pm 35.37$ & $20.44 \pm 3.67$ \\
\hline
\end{tabular}

Table 2: The serum levels of Fasting blood glucose levels of Group A and other groups in the study.

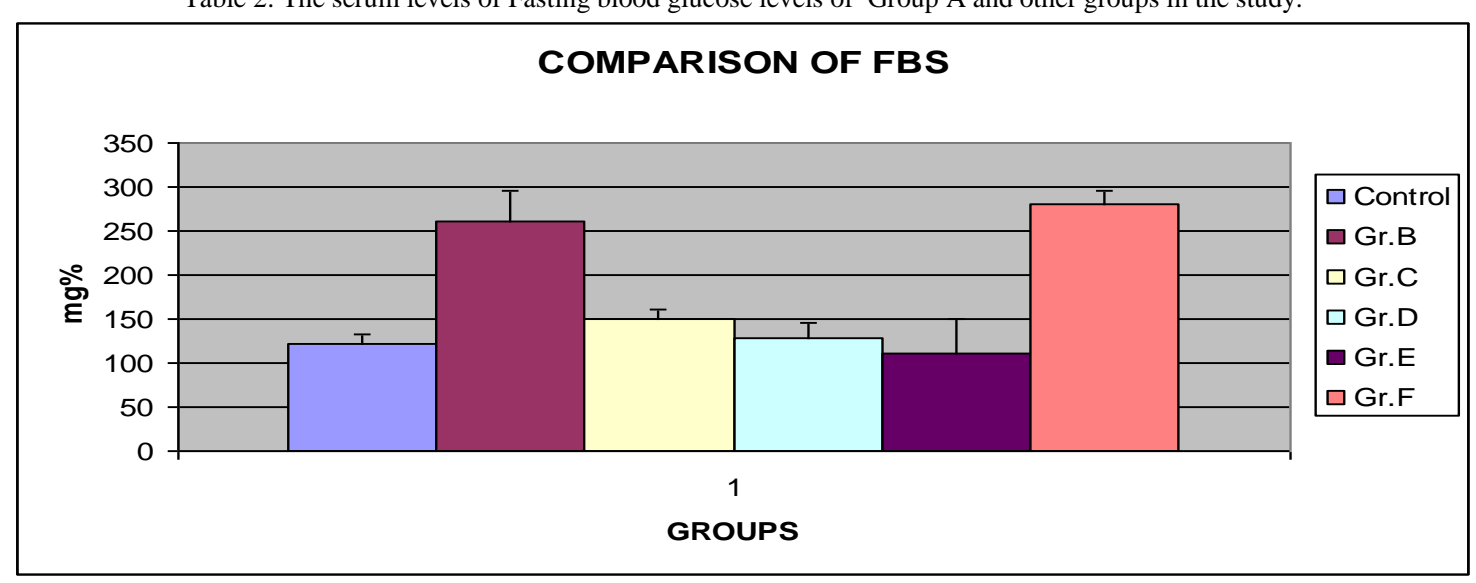

\begin{tabular}{|l|l|l|l|l|l|}
\hline FBS (mg \%) & & & & & \\
\hline Control & Gr.B & Gr.C & Gr.D & Gr.E & Gr.F \\
\hline $121 \pm 10.82$ & $260.77 \pm 35.33$ & $149.5 \pm 11.69$ & $127.98 \pm 16.81$ & $110.68 \pm 40.35$ & $280.77 \pm 15.44$ \\
\hline
\end{tabular}

\section{Discussion}

Escalating data in both investigational and scientific studies advocates that oxidative stress plays a key role in the pathogenesis of both types of diabetes mellitus. Free radicals are produced inexplicably in diabetes by glucose oxidation, nonenzymatic glycation of proteins, and the subsequent oxidative degradation of glycated proteins. Unusual high levels of free radicals and the concurrent decline of antioxidant defense system causes dent to cellular organelles and enzymes, increased lipid peroxidation, and development of insulin resistance. These consequences of oxidative stress can endorse the impediment of diabetes mellitus. During this vivo study of the effects of various conventional and alternative drugs on various biomarkers are assessed. There is a need to continue to explore the relationship between free radicals, diabetes, and its complications, and to elucidate the mechanisms by which increased oxidative stress accelerates the development of diabetic complications, in an effort to expand treatment options.

Some plants and trace elements may be proven the candidates to bring about the promising and desirable results to circumvent the day-by-day spreading menace of diabetes mellitus [10]. Coriander has been documented as a traditional 
treatment for diabetes Mellitus. Similarly Vanadyl sulfate exhibit the anti diabetic activity yet its toxicity have been observed at high doses

In plants, mineral uptake is the process in which minerals enter the cellular material, typically following the same pathway as water transport. The most normal entrance portal for mineral uptake is through plant roots [28]. While up taking the minerals, the toxicity is detoxified probably by the process of auto-oxidation in plants. Keeping this hypothesis in our minds, we tried to get the Vanadyl sulfate uptaken by coriander to make it detoxified. This has made the toxic Vanadyl sulfate non-toxic and living being consumable for producing antidiabetic affects without deleterious effects.

All the available data and studies, until now, are showing their hypoglycemic effects but no one has reported their combined activity, the appropriate doses and/or their side/after effects and mechanism of action while treating Diabetes Mellitus. The Coriandrum Sativum and Vanadyl sulfate separately or in combination may protect the complications of DM. These complications may be forestaller through up regulation or down regulation of Superoxide Dismutase, Catalase and Glutathione Peroxidase. There is still to explore the mechanism of Coriandrum Sativum and Vanadyl sulfate in DM through the different antioxidant enzyme system.

This study is a two-dimensional study that revolves around two aspects i.e. one it evaluates the antidiabetic and nontoxic triumphs of the ameliorated affects of the Vanadyl sulfate up taken Coriander and the other, possible autooxidation of Vanadyl sulfate through coriander.

Whereas observing the results of the fasting blood glucose levels of STZ induced diabetes in albino rats, the group B (Diabetic rats treated with Vanadyl sulfate only)showed significant rise i.e. 53.5\% in FBS while compared with Control group (Group A), though they were treated with Vanadyl sulfate. This result indicative that Vanadyl sulfate did not affect the FBS levels of the STZ induced diabetes in albino rats. These results are in agreement of Gail R [29] but do not favor a study conducted in France [20]. They established the affective role of Vanadyl sulfate on FBS.

While comparing the FBS levels of group C (Diabetic rats treated with coriander only) with the group A (Control), 19\% increase in FBS has been noted that was not at par with the control group. It reflects that the antidiabetic treatment with coriander only may decrease the FBS level but still the required normal range was not achieved. Same study conducted [29] in 2011, showed some how the matched results [30] but the present study results are not in agreement with another medical cram[31] because it showed no effect of coriander on the FBS level.

When the FBS level of the group D (Diabetic rats treated with Vanadyl sulfate enriched coriander) was weigh against Control group, only 5\% difference was detected. It was pinpointing that the augmented antidiabetic effect of the Vanadyl sulfate enriched coriander lowered the FBS level. Statistically the probability is $>0.01$ of group D, that is highly corresponding to control group.

In comparison of the group E (diabetic rats treated with Insulin only) with control almost the same FBS level was obtained. This was obvious as there is established role of insulin for lowering the blood glucose. However, when the same group is compared with group D (Diabetic rats treated with Vanadyl sulfate enriched coriander) it relatively managed the FBS level in the same manner as of the group D (only 15\% higher). Thus, it may be assumed that treating IDDM with Vanadyl sulfate up taken coriander, may exhibit same results as of the effects produced by the insulin while lowering the FBS. Comparison of group F (D+ No Treatment) with the control illustrates no control on FBS level and it was in contrast with group B $(\mathrm{D}+\mathrm{V})$, that demonstrated the same results.

Total antioxidant status or Total Antioxidant Capacity within a matrix should reflect the collective contribution to reducing properties of the individual antioxidants or electron-donating components [32]. Compared with the control groups the Total Antioxidant Capacity was depleted in diabetes. Decreased activity of the antioxidant enzymes and depletion of total antioxidant capacity may increase the susceptibility of diabetic patients to oxidative injury. In the light of these facts a significant correlation, between total antioxidant capacity and clinical characteristics of diabetic patients including their blood levels of glucose and glycated hemoglobin, suggests that the measurement of total antioxidant capacity in diabetic patients can be a marker of glycaemic control [5].

Therefore, the assessment of TAC in our study is of particular consideration. Our control group (Group A) of the rats exhibited $193.88 \mu \mathrm{mol} / \mathrm{L}$ of TAC serum level. When it is compared to group B (D+V), it reveals that the TAC level in the blood fell down to $55.6 \%$ in the group that was treated only by Vanadyl sulfate. This result defines that Vanadyl sulfate did not affect the serum TAC levels when given to the diabetic rats. This finding statistically has exceptional dissimilarity with control group ( $\mathrm{p}<0.001)$. Some researchers are in support to the results of our study and their studies describe that the Vanadyl sulfate has no role on TAC [33] However, in 2010, [34] a study, unveil that TAC could be taken back to normal level by Vanadyl sulfate while caring for DM.

Comparison of Group C (Diabetic rats treated with coriander only) with the Control group showed that $45.0 \%$ fall had been noticed in TAC level of the group $\mathrm{C}$ than Control group. Though, these are somehow better fallout than that of the results of Group B yet the coriander was unable to bring back the TAC up to desired level. This may be because that the glycemic control was poorer by the coriander and thus the TAC remained far decreased from the standard echelon. A few Scientists [35] produced different outcomes from our mentioned results. Conversely, majority disclosed the similar consequence. In 2012, [36] a scientific study produced matched conclusions, as that of ours. 
Group D (Diabetic rats treated with Vanadyl sulfate uptaken Coriander) findings for TAC are very attention grabbing. In contrast to the Group A, group D reveals only 5\% less Total Antioxidant Capacity. It interprets that treating STZ induced diabetes in albino rats with Vanadyl sulfate up taken coriander not only control hyperglycemia but it can maintain TAC in DM too. This might be due to the reason that when sugar is kept under control limits it minimizes the oxidative stress there keeping SOD levels close to normal ranges and therefore TAC also matches the control.

When the group E (diabetic rats treated with Insulin only) was compared for TAC, with the control group (group A), 3\% fall was observed in the STZ induced diabetes in albino rats treated with Insulin. This was an expected result as the roll of Insulin is well recognized in treating DM [37]. Keeping our finding regarding this parameter, it can be said that Insulin treatment definitely controls the blood sugar level but also maintains the TAC.

Interestingly, these above mentioned results at the same time judged against the finding of Group D (Diabetic rats treated with Vanadyl sulfate uptaken Coriander) merely $2.2 \%$ difference was noted between the groups treated with Vanadyl sulfate uptaken Coriander and Insulin. This is conclusive that treating STZ induced diabetes in albino rats with Vanadyl sulfate up taken coriander may insulinomimetic effects. Also it may be due to the e described as previously that Vanadyl sulfate up taken coriander successfully controlled the FBS of the STZ induced diabetes in albino rats, This also effectively improve the vitamin $\mathrm{D}$ and thus demonstrated antioxidant effect.

Another obvious finding was found in comparing the Group F (D+ No treatment) with control group. It is very clear to observe that the serum TAC is very much decreased i.e. $84.8 \%$ of the control due to the decreased antioxidant level. The values are highly significant ( $\mathrm{p}<0.001$ ), when compared to serum TAC level of the Group. Latest and contemporary studies also provided comparative results as to our findings [38]. On the contrary, [39] another finding varies our results. Possibly it may be due to the shorter duration of his study and the numbers of subjects were also fewer.

Our discussion emphasizes the insulinomimetic activity of the Vanadyl sulfate uptaken coriander sativum and its effects on TAC. It has been observed during the study that the diabetic rats of group D that were treated with the Vanadyl sulfate uptaken coriander sativum not only demonstrated the good glycaemic control but also exhibited Total AntiOxidant Capacity at par with the Control group (Group A) and in those rats as well, that were on insulin treatment (Group E). It is indicative that Vanadyl sulfate uptaken coriander sativum shows insulinomimetic activity along with commendable effects on TAC.

\section{Conclusion}

The present study agreements the efforts of scientific community for lessen the toxic effects of vanadyl sulfate as an insulinomimetic agent. For this purpose, a characteristic of auto-oxidation of trace elements through plants has been exploited in this study. In this regard the detoxification of vanadyl sulfate has been achieved through possible auto oxidation of the valuable trace elements by coriander.

The effect of vanadyl sulfate uptaken by coriander acts as antidiabetic agent and shows insulinomimetic and antioxidant activity in the treatment of Diabetic rats. After uptaken the vanadyl sulfate by coriander the results obtained in this study prompted us to conclude that in this method combined antidiabetic and antioxidant effect of the two, potentiates each other becoming much more than their individual effects.

\section{Acknowledgements}

The authors are thankful to Maxitech Pharma for their support in providing logistics to execute the research work.

\section{References}

[1] Sarah Wild, Gojka Roglic, Anders Green.Global Prevalence of Diabetes.Estimates for the year 2000 and projections for 2030.Diabetes Care 27:1047-1053, 2004.

[2] Mohsen Kerkeni, Amel Saidd, Hassan Bouzidi, Ahmed Letaief, Mohamed Hammami. Serum of advanced glycation end products in Tunisian diabetic patients with chronic kidney disease. International Journal of Diseases and Disorders Vol. 1 (1). 007-012, 2013.

[3] Jalil Momen-Beitollahi , Arash Mansourian, Fatemeh Momen-Heravi, Masoud Amanlou , Stoyana Obradov , Mahnaz Sahebjamee Assessment of salivary and serum antioxidant status in patients with recurrent aphthous stomatitis. Med Oral Patol Oral Cir Bucal. Jul 1; 15 (4):e557-61. 2010.

[4] Alba Naudi, Mariona Jove, Victoria Ayala, Anna Cassanye, Jose Serrano, Hugo Gonzalo, Jordi Boada, Joan Prat, Manuel Portero-Otin,, Reinald Pamplona. Cellular Dysfunction in Diabetes as Maladaptive Response to Mitochondrial Oxidative Stress. Experimental Diabetes Research Article ID 696215, Volume 2. 1-14. 2012

[5] Wan Ting Hsu,Li Yu Tsai, Shu Kai Lin, Jen Kuei Hsiao, and Bai Hsiun Chen.Effects of diabetes duration and glycemic control on free radicals in children with type 1 diabetes mellitus. Annuals of Clinical \& Laboratory Science, 36( 2): 2006 
[6] David M. Nathan, Mdjohn B. Buse,Mayer B. Davidson, Robert J. Heine, Rury R. Holman, Robert Sherwin,Bernard Zinman.Management of hyperglycemia in type 2 diabetes: A consensus algorithm for the initiation and adjustment of therapy a consensus statement from the American Diabetes Association and the European Association for the study of diabetes.Diabetes care, 29( 8), 2006.

[7] Esra Saatci, Gulruh Tahmiscioglu, Nafiz Bozdemir, Ersin Akpinar, Sevgi Ozcan and Hatice Kurdak.Reseaerch well-being and treatment satisfaction of diabetic patients in primary care. Health and Quality of Life Outcomes, 8:67, 2010.

[8] Hong Fang Song, Wenbo Jia, Ying Yao, Yafei Hu, Lin Lei, Jie Lin, Xiufa Sun and Liegang Liu.Oxidative stress, antioxidant status and DNA damage in patients with impaired glucose regulation and newly diagnosed Type 2 diabetes. Clinical Science 112, 599-606, 2007.

[9] Padavala Ajay Babu, Gadde Suneetha, Radha Boddepalli, Vedurupaka Vasantha Lakshmi, Talluru Sudha Rani, Yellapu RamBabu and Kolli Srinivas. A database of 389 medicinal plants for diabetes.Bioinformation 1(4): 130-131,2006

[10] Gholamali Jelodar, Maleki Mohsen and Sirus Shahram.Effect of Walnut Leaf, Coriander and Pomegranate on blood glucose and histopathology of pancreas of Alloxan induced diabetic rats. Research Paper: African Journal of Traditional, Complimentary and Alternative Medicines, 4(3):299-305, 2007

[11] Williams, Patricia A.M, Baran, Enrique J. Vanadium detoxification: On the interaction of oxovanadium (IV) and other vanadium species with 2,3-dimercapto-1-propanesulfonate. Journal of Inorganic Biochemistry, Volume 102, issue 5-6. 1195-1198. 2008

[12] G.B. Kavishankarl, N. Lakshmidevi1, S. Mahadeva Murthy, H.S. Prakash,S.R. Niranjana. Diabetes and medicinal plants-A review. Int J Pharm Biomed Sci 2(3), 65-80. 2011

[13] Ebtesam A. Al -Suhaimi. Effect of Coriandrum sativum, a common herbal medicine, on endocrine and reproductive organ structure and function. The Internet Journal of Alternative Medicine. Volume 7 Number 2 ISSN: 1540-2584. 2009

[14] Subodh Verma , Margaret C. Cam and John H. McNeill. Nutritional Factors that Can Favorably Influence the Glucose/Insulin System: Vanadium. Journal of the American College of Nutrition, Vol. 17, No. 1, 11-18. 1998.

[15] D.M. Smith, R.M. Pickering and G.T. Lewith. A systematic review of vanadium oral supplements for glycaemic control in type 2 diabetes mellitus. Q J Med, 101:351-358, 2008

[16] B. Ramachandran, D. Sathish Sekar, M. Kandaswamy, V. Narayananand S. Subramanian.Experimental hypoglycemic effect of macrocyclic binuclear Oxovanadium (IV) complex on Streptozotocin-Induced diabetic rats. Diab. Res., 5:137-142, 2004

[17] Catherine 1. Martin, james albers, william h. Herman, patricia cleary, barbara waberski, douglas a. Greene, martin j. Stevens, eva 1. Feldman. Neuropathy among the diabetes control and complications trial cohort eight years after trial completion. Diabetes care. Vol. 29, no. 2, 340344. 2006

[18] Stephanie J. Garcia, Kristin Gellein, Tore Syversen, and Michael Aschner. Iron deficient and manganese supplemented diets alter metals and transporters in the developing rat brain. Toxicological Sciences 95(1), 205-214,2007

[19] Z. Durackova. Some Current Insights into Oxidative Stress. Physiol Res. 59: 459-469. 2010

[20] K. Cusi, S. Cukier, R. A. Defronzo, M. Torres, F. M. Puchulu and J. C. Pereira Redondo. Vanadyl Sulfate improve hepatic and muscle Insulin sensitivity in type 2 diabetes. J Clin Endocrinol Metab 86:1410-1417, 2001

[21] Alessandro Alimonti, Francesco Petrucci, Michael Krachler, Beatrice Boccaa and Sergio Carolia.Reference values for chromium, nickel and vanadium in urine of youngsters from the urban area of Rome. J. Environ. Monit ,2, 351-354, 2000

[22] Atsuko Adachi, Kaoru Asai, Yukari Koyama,Yumiko Matsumoto, and Toshio Okano. Subacute Vanadium toxicity in rats. Journal of Health Science, 46(6) 503-508,2000

[23] E. G. Ferrer, E. J. Baran. Reduction of vanadium(V) with ascorbic acid and isolation of the generated oxovanadium (IV) species. Jour Biological Trace Element Research. Volume 83, Issue 2. 111-119. 2001

[24] Nakagawa T, Sato W, Glushakova O, Heinig M, Clarke T, Campbell-Thompson M, Yuzawa Y, Atkinson MA, Johnson RJ, Croker B. Diabetic endothelial nitric oxide synthase knockout mice develop advanced diabetic nephropathy. AMDCC (Animal Models of Diabetic Complications Consortium).2005. J Am Soc Nephrol 18:539-50. 2007

[25] Iris F. F. Benzie, 1 and J. J. Strain. The Ferric Reducing Ability of Plasma (FRAP) as a Measure of "Antioxidant Power": The FRAP Assay. Department of Health Sciences, Hong Kong Polytechnic University, Hung Hom, Kowloon, Hong Kong; and Human Nutrition Research Group, University of Ulster, Coleraine, Northern Ireland, United Kingdom. Analytical biochemistry Article no. 0292, 239: 70 - 76.1996.

[26] B Deepa and CV Anuradha. Antioxidant potential of Coriandrum Sativum seed extract. Indian Journal of Experimental Biology, 49:30-38, 2011

[27] Megha Saraswat, Palla Suryanarayana,Paduru Yadagiri Reddy, Madhoosudan A. Patil,Nagalla Balakrishna,Geereddy Bhanuprakash Reddy.Antiglycating potential of Zingiber officinalis and delay of diabetic cataract in rats. Molecular Vision; 16:1525-1537, 2010

[28] Rajeshwari C U and Andallu B. Oxidative stress in NIDDM patients: Influence of coriander (Coriandrum sativum) seeds, Research Journal of Pharmaceutical, Biological and Chemical sciences. 2(1):31-41, 2011

[29] Menakshi Bhat, Smita S. Zinjarde, Shobha Y. Bhargava, Ameeta Ravi Kumar and Mi-Ae Bang, Hyeon-A Kim and Young-Ja Cho.Alterations in the blood glucose, serum lipids and renal oxidative stress in diabetic rats by supplementation of onion (Allium cepa. Linn). Nutrition Research and Practice, 3(3), 242-246, 2009

[30] Gail R. Willsky, Lai-Har Chi, Yulan Liang, Daniel P. Gaile, Zihua Hu, and Debbie C. Crans. Diabetes-altered gene expression in rat skeletal muscle corrected by oral administration of vanadyl sulfate. Physiol. Genomics 26:192-201, 2006

[31] K Sushruta, S Satyanarayana, N Srinivas, J Raja Sekhar. Evaluation of the Blood-Glucose Reducing Effects of Aqueous Extracts of the Selected Umbelliferous Fruits Used in Culinary Practices. Tropical Journal of Pharmaceutical Research. 5 (2): $613-617.2006$

[32] M. E. Rahbani-Nobar, A.Rahimi-Pour, M.Rahbani-Nobar, F.Adi-Beig, S.M.Mirhashemi. total antioxidant capacity superoxide dismutase and glutathione peroxidase in diabetec patients. Medical Journal of Islamic Academy of Sciences 12:4, 109-114,1999

[33] Hazem A. H. Kataya and AlaaEldin A. Hamza.Red Cabbage (Brassica oleracea) Ameliorates Diabetic Nephropathy in Rats. Original Article, ;5(3)281-287, 2007

[34] Gurjeet S. Birdee, and Gloria Yeh. Complementary and Alternative Medicine Therapies for Diabetes: A Clinical Review. Clinical Diabetes. Volume 28, Number 4, $1-3.2010$

[35] Resat A, Kubilay G, Birsen D. Comparative evaluation of various total antioxidant assays applied to phenolic components with the CUPRAC assay. Mol.12:1496-1547. 2007

[36] Jaakko Tuomilehto, Jaana Lindstrom, Johan G Eriksson, Timo T Valle, Helena Hamalainen, Pirjo Ilanne Parikka, Sirkka Keinanen Kiukaanniemi, Mauri Laakso, Anne Louheranta, Merja Rastas, Virpi Salminen, Matti Uusitupa. Prevention of Type 2 diabetes mellitus by changes in lifestyle among subjects with impaired glucose tolerance. N Engl J Med, Vol. 344, No. 18; $1343-1350.2001$

[37] Wan Ting Hsu,Li Yu Tsai, Shu Kai Lin, Jen Kuei Hsiao, and Bai Hsiun Chen. Effects of diabetes duration and glycemic control on free radicals in children with type 1 diabetes mellitus. Annuals of Clinical \& Laboratory Science, 36(2): 174 - 178.2006

[38] Pallab Das Gupta , Amartya De. Diabetes Mellitus and its Herbal Treatment. International Journal of Research in Pharmaceutical and Biomedical Sciences. Vol. 3 (2); $706-721.2012$ 
[39] Eleazu Chinedum. O. Okafor, Polycarp N, Amajor John, Awa Ezinne, Ahamefula I, Eleazu Kate C. Chemical Composition, antioxidant activity, functional properties and inhibitory action of unripe plantain (M. Paradisiacae) flour. African Journal of Biotechnology, Vol. 10 (74), 16948-16952. 2011

[40] Iyer UM, Desai PA, Venugopal S. Int J Green Pharm. Impact of panchratna juice in the management of diabetes mellitus: Fresh vs. processed product. Vol: 4 (2); 122-128. 2010 